\title{
Wigner-Ville Spectral Analysis of Nonstationary Processes
}

\author{
WOLFGANG MARTIN AND PATRICK FLANDRIN
}

\begin{abstract}
The Wigner-Ville spectrum has been recently introduced as the unique generalized spectrum for time-varying spectral analysis. Its properties are revised with emphasis on its central role in the analysis of second-order properties of nonstationary random signals. We propose here a general class of spectral estimators of the Wigner-Ville spectrum: this class is based on arbitrarily weighted covariance estimators and its formal description corresponds to the general class of conjoint time-frequency representations of deterministic signals with finite energy. Classical estimators like short-time periodograms and the recently introduced pseudo-Wigner estimators are shown to be special cases of the general class. The generalized framework allows the calculation of the moments of general spectral estimators and comparing the results emphasizes the versatility of the new pseudo-Wigner estimators. The effective numerical implementation, by an $N$-point FFT, of pseudo-Wigner estimators of $2 \mathrm{~N}$ points is indicated and various examples are given.
\end{abstract}

\section{INTRODUCTION}

$\mathrm{D}$ ESPITE recent interest in (and development of) the time domain modeling of random signals, spectral analysis is still of fundamental importance in the study of stationary, linear processes. This is true for several reasons, mainly [1],

- the immediate physical interpretation of the spectrum is a power frequency distribution,

- the spectrum is the basis for (and starting point of) time domain modeling,

- the spectrum plays a central role in linear prediction and filtering,

- the spectrum can be calculated rather efficiently by adequate FFT techniques.

But, in many applications, the assumption of stationarity fails to be true: the physical character of random signals demands a nonstationary approach such as in acoustics, speech, geophysics, biology, biomedicine fields, etc. However, a spectrum of nonstationary processes cannot be defined by simply generalizing the ordinary stationary spectrum. Loynes [2] has discussed desired properties of a spectrum of nonstationary processes: these properties are known to be a set of contradicting properties. In particular, having a spectrum with nonnegative values ex-

Manuscript received February 17, 1984; revised January 29, 1985 and July 8, 1985. The work of W. Martin was supported by the Deutsche Forschungsgemeinschaft under Grant Ma 895/2.

W. Martin is with the Botanisches Institut der Universität Bonn, Kirschallee 1, D-5300 Bonn 1, Germany.

P. Flandrin is with the Laboratoire de Traitement du Signal, LA 346 CNRS, ICPI, 69288 Lyon Cedex 02, France, and GRECO 69 "Systèmes adaptatifs." cludes compatibility with linear filtering and modulation [3].

Nevertheless, one has tried to estimate a kind of "timevarying" spectrum by performing a locally stationary spectral analysis. The most popular technique to do so is the short-time periodogram analysis [4].

The approach by short-time periodograms brings up the difficulty that the estimated time-varying spectra depend on the choice of the used data window. Priestley [5] tried to overcome this problem by his evolutionary spectra of oscillatory processes. Evolutionary spectra retain the nonnegativity property of the ordinary spectrum and they can be defined for time-varying linear models like time-dependent autoregressive models [6], [7], but for a given nonstationary process, there is no unique evolutionary spectrum and, in general, the "frequency variable" cannot be interpreted as frequency defined by the time-frequency duality of stationary processes [8]. Recently, a new theoretical concept of a conjoint time-frequency representation has been developed for deterministic signals [9], [10] which could be carried over to harmonizable random signals, a quite wide class of nonstationary processes [11]. Sacrifying the nonnegativity property of a time-varying spectrum, it could even be shown that the resulting Wigner-Ville spectrum is the unique solution which can be obtained in generalizing the classical ordinary spectrum under "natural conditions" [12]. The new theoretical approach to spectral analysis of nonstationary processes inplies the construction of new estimators called pseudoWigner estimators [13], [14]. We shall now develop their statistical properties and consider competing estimators like short-time periodograms. Practical design rules and applications will also be included.

\section{Conjoint Time-Frequency Representations of Harmonizable Processes}

\section{A. Harmonizable Processes}

Harmonizable processes have been shown to be candidates for a time-varying spectral analysis [15]. This concept is not only necessary from a theoretical point of view, but also most of the measured random signals enter this large subclass of nonstationary processes. Actually, the only requirement relies on the existence of a bidimensional Fourier-Stieltjes transform $f$ of the autocovariance function $K$ [16]: 


$$
\begin{aligned}
K\left(t_{1}, t_{2}\right) & =E\left\{X\left(t_{1}\right) X^{*}\left(t_{2}\right)\right\} \\
& =\frac{1}{4 \pi^{2}} \iint_{-\pi}^{\pi} e^{i\left(\lambda t_{1}-\mu t_{2}\right)} f(\lambda, \mu) d \lambda d \mu .
\end{aligned}
$$

In (1), $X(t), t=\cdots,-1,0,1, \cdots$ is a centered complex-valued random process with existing second moments. By $E$, we denote the expectation operator and the star indicates complex conjugation.

We can interpret in (1) the autocovariance function $K$ as a time versus time representation of the second-order properties of $X(t)$ and the "spectral density function" $f$ as a frequency versus frequency representation which are linearly connected by the Fourier transformation. We may then very intuitively postulate an intermediate time-frequency spectrum $W$. In [11], it has been shown that an adequate choice of $W$ is given by the Wigner-Ville spectrum:

$$
W(t, \omega)=\frac{1}{\pi} \int_{-\pi}^{\pi} e^{i 2 \zeta t} f(\omega+\zeta, \omega-\zeta) d \zeta
$$

which leads to

$$
2 K(t+\tau, t-\tau)=\frac{1}{2 \pi} \int_{-\pi}^{\pi} e^{i \omega \tau} W\left(t, \frac{\omega}{2}\right) d \omega .
$$

If we can invert (3), an equivalent definition of the Wigner-Ville spectrum is given by

$$
W(t, \omega)=2 \sum_{\tau=-\infty}^{\infty} K(t+\tau, t-\tau) e^{-i 2 \omega \tau} .
$$

These are the discrete-time relations analogous to the discrete Wigner representation of deterministic signals introduced by Claasen and Mecklenbräuker [9] since (4) can be written in the form [11]

$$
W(t, \omega)=E\left\{2 \sum_{\tau=-\infty}^{\infty} X(t+\tau) X^{*}(t-\tau) e^{-i 2 \omega \tau}\right\} .
$$

The introduction of this distribution goes back to Wigner [17] in quantum mechanics and the pioneering work of Ville [18] in signal theory. It should be mentioned that this approach has empirically been used by other authors too in order to define a time-varying spectral analysis (cf. [19] and [20]), but one noticed neither the connection to the general time-frequency dualism of nonstationary linear processes nor the attracting properties of this approach, nor the possibility to prove its unicity under appropriate conditions. This is followed up in the next section.

\section{B. Properties of the Wigner-Ville (WV) Spectrum}

In the case of deterministic signals with finite energy, a list of the properties of the Wigner-Ville representation is given in [9], for instance. These properties carry over to the case of harmonizable processes [11]. Here, we therefore list only properties which are needed in the following.

1) Transformation properties: See (2) and (3).
2) Marginal properties:

$$
\begin{aligned}
\operatorname{Var}\{X(t)\} & =\frac{1}{2 \pi} \int_{-\pi / 2}^{\pi / 2} W(t, \omega) d \omega \\
f(\omega, \omega)+f(\omega+\pi, \omega+\pi) & =\sum_{t=-\infty}^{\infty} W(t, \omega) .
\end{aligned}
$$

Equation (7) shows one difficulty of the discrete version of the Wigner representation: in the frequency variable, it is periodic with period $\pi$ instead of $2 \pi$. This may be avoided if we oversample the signal by a factor of at least two or if we use analytic signals instead of real-valued signals.

3) Translation properties:

if $Y(t):=X(t-\tau)$, then $W_{Y}(t, \omega)=W_{X}(t-\tau, \omega)$;

if $Y(t):=X(t) e^{i \omega t}$, then $W_{Y}(t, \omega)=W_{X}\left(t, \omega-\omega_{0}\right)$.

4) Local moment properties:

$$
K(t+1, t-1)=\frac{1}{2 \pi} \int_{-\pi / 2}^{\pi / 2} e^{i 2 \omega} W(t, \omega) d \omega
$$

$$
\begin{array}{r}
-\left.\frac{1}{2}\left\{\frac{\partial}{\partial \omega} f(\omega, \zeta)+\frac{\partial}{\partial \omega} f(\omega+\pi, \zeta+\pi)\right\}\right|_{\zeta=\omega} \\
=\sum_{t=-\infty}^{\infty} t W(t, \omega)
\end{array}
$$

supposing the expressions on the left-hand side exist.

Properties (10) and (11) can be used to define the instantaneous frequency and group delay for nonstationary, but harmonizable processes [11].

5) Negativity: The WV spectrum is always real-valued, but can very well take on negative values. Therefore, it does not give a "true" density.

6) Interference: There exist interference effects between structures in the time-frequency plane due to the bilinearity of the definition (4). Such interference phenomena can be removed by using a pseudo-WV spectrum which is an appropriately smoothed version of the WV spectrum (cf. [21]).

7) Unicity: The WV spectrum is the only time-varying spectrum which

- reduces to the ordinary spectral density if the process is stationary and satisfies the concepts of

- linear time-frequency dualism,

- linear filtering and modulations together with a proper interpretation of local moments [cf. 4)] [12].

These properties show that the WV spectrum suffers only from the disadvantage of attaining negative values. All the other properties favor the WV spectrum and, furthermore, the condition of nonnegativity contradicts the 
other desirable properties [3] which seem more important than the mere interpretation as a true density. However, the consequence of such a choice is twofold. First, the use of the WV spectrum requires a careful interpretation which naturally excludes any local energy signification (see, e.g., the discussion in [29]). Second, it will appear in the next section that the smoothing out of negative values by appropriate averaging procedures is closely related to a practical estimation of the WV spectrum.

This problem of estimation will now be addressed, keeping in mind that the quantity to estimate is precisely and uniquely defined. This was the necessary prerequisite for any estimation procedure since some of the possible coming up estimators have yet been considered, but aimed at different time-varying spectra (e.g., Priestley's evolutionary spectra).

\section{Estimation of the Wigner-Ville Spectrum}

\section{A. Quasi-Stationary, Analytic, Gaussian Processes}

A common problem in analyzing random signals consists of the unavailability of ensemble averages. By assuming ergodicity, ensemble averages can be replaced by time averages, but for nonstationary processes, this seems impossible since constructing a time average would smooth out any time-varying property of the signal.

To overcome this difficulty, we shall therefore use a concept of quasi-stationarity. We shall suppose that the autocovariance function $K$ undergoes a "slow evolution," i.e., given a time instant $t$, there exists a stationary autocovariance function $K_{t}$ and an interval $T$ on which we have

$$
\left|K(t+\tau, t-\tau)-K_{t}(2 \tau)\right|<\epsilon(T)
$$

and where $\epsilon(T)$ is a measure of the approximation. As time of stationarity $T_{s}$ we call the smallest $T$ for which (12) holds. A nonstationary process is called quasi-stationary if $T_{s}>0$ for a given $\epsilon>0$.

In order to derive the moments of the estimators to be discussed here, we shall assume that $X(t)$ is a Gaussian process. Furthermore, we will only consider real-valued signals, but complexify them by choosing their Hilbert transform as the imaginary part: this is analogous to passing to the analytic signal in the case of deterministic signals [16]. Therefore, we shall call these processes analytic processes. There are several advantages in dealing only with analytic processes.

- Instantaneous frequency of real-valued signals [cf. (10)] is only meaningful if passing to the associated analytic signals [22].

- Information on negative frequencies of any spectrum of a real-valued signal is redundant.

- Aliasing in the discrete version of the Wigner distribution is avoided [9], as well as interference terms between positive and negative frequencies.

- Calculations of moments of the estimators are more straightforward.

Now, if we want to realize the estimators, one usually will use digital signal processing. Therefore, we shall only treat the discrete-time case.

\section{B. A General Class of Spectral Estimators}

In discrete time, the Wigner-Ville spectrum is the discrete Fourier transform of the autocovariance function as given by (4). The most general estimator of the WignerVille spectrum relies, therefore, on general covariance estimators of the type [23]

$$
\begin{aligned}
\hat{K}(t+k, t-k):= & \sum_{m=-\infty}^{\infty} \Phi(m, 2 k) X(t+m+k) \\
& \cdot X *(t+m-k) .
\end{aligned}
$$

In (13), $\Phi(m, 2 k)$ is an arbitrary data window determining the kind of averaging to be performed on the products $X(t$ $+m+k) X^{*}(t+m-k)$ in order to estimate $K$. Assuming that $\Phi$ has a discrete Fourier inverse $\phi$, i.e.,

$$
\Phi(m, 2 k)=\frac{1}{2 \pi} \int_{-\pi}^{\pi} \phi(n, 2 k) e^{i n m} d n,
$$

and inserting (14) in (13) and (13) in (4), a class of estimators of the Wigner-Ville spectrum is given by

$$
\begin{aligned}
\hat{W}(t, \omega ; \phi)= & \frac{1}{\pi} \sum_{k=-\infty}^{\infty} \sum_{m=-\infty}^{\infty} \int_{-\pi}^{\pi} e^{i n m} \phi(n, 2 k) \\
& \cdot X(t+m+k) X^{*}(t+m-k) \\
& \cdot e^{-i 2 \omega k} d n .
\end{aligned}
$$

Equation (15) is exactly the discrete-time version of the general conjoint time-frequency representation of deterministic signals with finite energy, as introduced in quantum mechanics by Cohen [24] and in signal processing by Escudié and Gréa [25]. Therefore, we consider (15) as the most general class of spectral estimators. Since the Wigner-Ville spectrum of stationary signals reduces to the ordinary spectrum, this class of estimators is valid for both stationary and nonstationary random signals.

The properties of general conjoint time-frequency representations have been extensively discussed [9], [10]; we can therefore immediately use these results and indicate the most important properties of some special estimators. However, new normalizations of $\phi$ are required since we have to carry over the finite energy case to the finite power case.

In the following, we first calculate the moments of general spectral estimators, and we then evaluate special cases.

\section{Moments of General Spectral Estimators}

A general expression of the first moment of the general spectral estimator can be derived from (15):

$$
\begin{aligned}
E\{\hat{W}(t, \omega ; \phi)\}= & \frac{1}{2 \pi} \sum_{m=-\infty}^{\infty} \int_{-\pi / 2}^{\pi / 2} W(m, \zeta) \\
& \cdot \Pi(m-t, \omega-\zeta) d \zeta
\end{aligned}
$$

where

$$
\Pi(t, \omega):=2 \sum_{k=-\infty}^{\infty} \Phi(t, 2 k) e^{-i 2 \omega k}
$$


Generally, this estimator is doubly biased in both time and frequency. The amount of bias in both directions depends on the weighting data window $\phi$ : hence, bias can be controlled by an appropriate design of $\phi$. For a proper normalization, however, i.e.,

$$
\frac{1}{2 \pi} \sum_{t=-\infty}^{\infty} \int_{-\pi / 2}^{\pi / 2} \Pi(t, \omega) d \omega=1,
$$

we get the condition

$$
\phi(0,0)=1 .
$$

The second moments of the general spectral estimator will be calculated by first using our assumption that $X(t)$ is a Gaussian and analytic random signal (Section III-A). This avoids the appearance of higher order cumulants in the expressions to be derived. We get

$$
\begin{aligned}
C:= & \operatorname{Cov}\left\{\hat{W}\left(t_{1}, \omega_{1} ; \phi\right), \hat{W}\left(t_{2}, \omega_{2} ; \phi\right)\right\} \\
= & 4 \sum_{k_{1}} \sum_{k_{2}} \sum_{m_{1}} \sum_{m_{2}} \Phi\left(m_{1}, 2 k_{1}\right) \Phi^{*}\left(m_{2}, 2 k_{2}\right) e^{-i 2\left(\omega_{1} k_{1}-\omega_{2} k_{2}\right)} \\
& \cdot K\left(t_{1}+m_{1}+k_{1}, t_{2}+m_{2}+k_{2}\right) \\
& \cdot K^{*}\left(t_{1}+m_{1}-k_{1}, t_{2}+m_{2}-k_{2}\right) .
\end{aligned}
$$

We now use our hypothesis of quasi-stationarity (12) and, as a final result, we get

$$
\begin{aligned}
C \sim & \frac{2}{\pi} \int_{-\pi}^{\pi} \sum_{k_{1}} \sum_{k_{2}} \exp \left[-i 2\left(k_{1}\left(\omega_{1}-v\right)\right.\right. \\
& \left.\left.-k_{2}\left(\omega_{2}-v\right)\right)\right] W_{\phi f_{t_{a}}, \phi f_{t, i}}(\tau, v) d v
\end{aligned}
$$

In (21), we have

$$
t_{a}=\frac{t_{1}+t_{2}}{2}+\frac{k_{1}+k_{2}}{2} ; \quad t_{b}=\frac{t_{1}+t_{2}}{2}-\frac{k_{1}+k_{2}}{2}
$$

and $f_{t}$ is the spectral density of the stationary process approximating $X(t)$ at $t . W_{\phi f t_{t}, \phi f_{t}}(\tau, v), \tau:=t_{1}-t_{2}$ is the cross-Wigner distribution of the windowed spectral densities at $t_{a}$ and $t_{b}$, i.e.,

$$
\begin{aligned}
W_{\phi f_{f_{l}}, \phi f_{t_{b}}}(\tau, v):= & \frac{1}{\pi} \int_{-\pi}^{\pi} e^{i 2 u \tau} \phi\left(2 u, 2 k_{1}\right) f_{t_{a}}(v+u) \\
& \cdot \phi^{*}\left(2 u, 2 k_{2}\right) f_{t_{i}}^{*}(v-u) d u
\end{aligned}
$$

The sign " " in (21) stems from the approximation of quasi-stationarity (12). Whereas (21) gives the general expression for the second moments of the general spectral estimator, it seems to be not very useful in practical applications. We can simplify (21) if we consider $\tau$ to be small enough such that

$$
K_{t_{a}}(\tau) \sim K_{t b}(\tau) \sim K_{t 0}(\tau), \quad t_{0}:=\left(t_{1}+t_{2}\right) / 2
$$

holds. Here, the cross-Wigner distribution reduces to the simple Wigner distribution, $t_{0}$ is no longer dependent on the summation of $k_{1}$ and $k_{2}$, and we get, starting with (21),

$$
\begin{gathered}
C \sim \frac{1}{2 \pi^{2}} \int_{-\pi}^{\pi} \int_{-\pi} \Psi\left(2 u, \omega_{1}-v\right) \Psi^{*}\left(2 u, \omega_{2}-v\right) \\
\cdot f_{t 0}(v+u) f_{t_{0}}^{*}(v-u) e^{-i 2 u \tau} d u d v
\end{gathered}
$$

with

$$
\Psi(u, v):=2 \sum_{k=-\infty}^{\infty} \phi(u, 2 k) e^{-i 2 k v} .
$$

The following diagram summarizes the relationships between the windows as expressed in the different time-frequency planes ( $F$ denotes discrete Fourier transform):

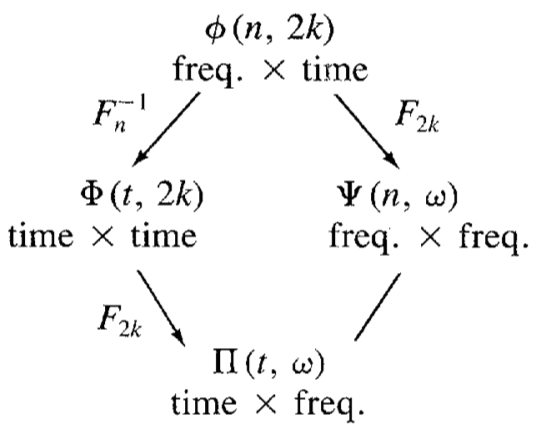

A further simplification can be achieved if we suppose that the time of stationarity [30] is much larger than the correlation time [1]. This ensures $\Psi(u, v)$ to be a peaked function in $u$ and (25) is led to the simplified expression [23]

$$
\begin{gathered}
C \sim \frac{1}{2 \pi} \sum_{t=-\infty}^{\infty} \int_{-\pi / 2}^{\pi / 2} \Pi\left(t-t_{1}, \omega_{1}-\omega\right) \\
\Pi *\left(t-t_{2}, \omega_{2}-\omega\right) f_{t_{0}}^{2}(\omega) d \omega .
\end{gathered}
$$

Equation (27) shows that the correlation between adjacent estimates only vanishes when the time-frequency windows $\Pi$ are nonoverlapping. Using (27), we finally get for the variance of the general estimator the expression

$$
\begin{aligned}
\operatorname{Var}\{\hat{W}(t, \omega ; \phi)\} \sim & \left\{\frac{1}{2 \pi} \sum_{t=-\infty}^{\infty} \int_{-\pi / 2}^{\pi / 2}\left|\Pi\left(t, \omega^{\prime}\right)\right|^{2} d \omega^{\prime}\right\} \\
& \cdot f_{t}^{2}(\omega) .
\end{aligned}
$$

From (28), it clearly appears that the approximated variance is ensured to be nonnegative.

\section{Short-Time Periodograms}

The most popular estimators of "time-varying spectra" are certainly short-time periodograms (cf. [4]). They are defined by

$$
J_{2 N-1}^{2}(t, \omega):=\frac{1}{2 N-1}\left|\sum_{k=-\infty}^{\infty} X(k) h_{N}(k-t) e^{-i \omega t}\right|^{2} .
$$

One also considers smoothed short-time periodograms, i.e.,

$$
J_{2 N-1}^{2,2 M-1}(t, \omega):=\sum_{m=-\infty}^{\infty} g_{M}(m) J_{2 N-1}^{2}(t+m, \omega) .
$$


In (29) and (30), $h_{N}$ and $g_{M}$ are windows with $2 N-1$, respectively $2 M-1$, nonzero values. Smoothed and unsmoothed short-time periodograms are special cases of the general spectral estimator: if one chooses

$$
\begin{aligned}
\phi_{\mathrm{STP}}(n, 2 k):= & \frac{1}{2 N-1} \sum_{j=-\infty}^{\infty} \\
& \cdot h_{N}(j+k) h_{N}^{*}(j-k) e^{\mathrm{inj}}
\end{aligned}
$$

then (15) reduces to (29). This weighting function has the form of an ambiguity function and we can denote

$$
\phi_{\mathrm{STP}}(n, 2 k)=: A_{h}(n, 2 k) .
$$

The smoothed short-time periodograms (30) result by a choice of

$$
\phi_{\mathrm{SSTP}}(n, 2 k):=\sum_{m=-\infty}^{\infty} g_{M}(m) e^{-i n m} \cdot A_{h}(n, 2 k) .
$$

The first moment of short-time periodograms can now be given by using (16), and we get

$$
\begin{aligned}
E\left\{J_{2 N-1}^{2,2 M-1}(t, \omega)\right\}= & \frac{1}{2 \pi} \frac{1}{2 N-1} \sum_{m=-\infty}^{\infty} \sum_{n=-\infty}^{\infty} \\
& \cdot g_{M}(m) \int_{-\pi / 2}^{\pi / 2} W(n+m, \zeta) \\
& \cdot W_{h}(t-n, \omega-\zeta) d \zeta
\end{aligned}
$$

where $W_{h}$ denotes the Wigner distribution of the data window $h_{N}$. The normalization condition (19) imposes on windows $g_{M}$ and $h_{N}$ to satisfy

$$
\frac{1}{2 N-1} \sum_{k=-\infty}^{\infty}\left|h_{N}(k)\right|^{2} \cdot \sum_{m=-\infty}^{\infty} g_{M}(m)=1 \text {. }
$$

Equation (34) expresses that even the unsmoothed shorttime periodogram $(M=1)$ is a doubly biased estimator of the Wigner distribution by the window $h_{N}$. The smoothed short-time periodogram $(M>1)$ undergoes a second bias contribution in the time direction due to the smoothing by the window $g_{M}$.

The second moments will only be stated if the approximation (24) holds, i.e., if $\tau=t_{1}-t_{2}$ is small enough. We can then start with (25); denoting

$$
H_{N}(u):=\sum_{k=-\infty}^{\infty} h_{N}(k) e^{-i k u},
$$

we can write (26) in the form

$$
\begin{aligned}
\Psi_{\mathrm{SSTP}}(2 u, v)= & \frac{1}{2 N-1} \sum_{m=-\infty}^{\infty} g_{M}(m) \\
& \cdot e^{-m i 2 u} H_{N}(v+u) H_{N}^{*}(v-u) .
\end{aligned}
$$

Then setting (37) in (25), we get

$$
\begin{aligned}
C \sim & \frac{1}{4 \pi^{2}(2 N-1)^{2}} \mid \sum_{m=-\infty}^{\infty} g_{M}(m) \int_{-\pi}^{\pi} f_{t 0}(\zeta) H_{N}\left(\omega_{1}-\zeta\right) \\
& \left.\cdot H_{N}^{*}\left(\omega_{2}-\zeta\right) e^{-i(\tau+m) \zeta} d \zeta\right|^{2}
\end{aligned}
$$

Under the reasonable assumption that the length of the window $h_{N}$ is large enough to ensure $H_{N}$ to be a peaked function, we obtain the final result:

$$
\begin{gathered}
C \sim f_{t_{0}}^{2}\left(\frac{\omega_{1}+\omega_{2}}{2}\right) \cdot \mid \sum_{m=-\infty}^{\infty} g_{M}(m) \\
\left.\cdot A_{h}\left(\omega_{2}-\omega_{1}, \tau+m\right)\right|^{2} .
\end{gathered}
$$

From this equation, it appears that both the smoothed and the unsmoothed short-time periodograms give estimates being correlated in both time and frequency. The correlation is described by the ambiguity function of the chosen window $h_{N}$. For a properly normalized window [cf. (35)], the variance of the short-time periodogram is given by

$$
\operatorname{Var}\left\{J_{2 N-1}^{2,2 M-1}(t, \omega)\right\} \sim f_{l}^{2}(\omega) \cdot\left|\sum_{m=-\infty}^{\infty} g_{M}(m) A_{h}(0, m)\right|^{2} .
$$

If $g_{M}$ is a rectangular window, the second term is of order $1 /(2 M-1)$ such that, as a crude approximation, we have

$$
\operatorname{Var}\left\{J_{2 N-1}^{2,2 M-1}(t, \omega)\right\} \sim \frac{1}{2 M-1} f_{t}^{2}(\omega) .
$$

Hence, short-time periodograms give doubly biased estimates of the Wigner-Ville spectrum due to the weighting function $\phi_{\text {STP }}$ which is an ambiguity function. This allows us to get rather smooth estimates, but refined signal structure is smoothed out. The variance of short-time periodograms decreases with the order of additional smoothing applied to the signal by the window $g_{M}(m)$. This induces a further decrease of resolution power for detection of refined structures.

\section{E. Pseudo-Wigner Estimators}

Recently, smoothed and unsmoothed pseudo-Wigner estimators have been introduced [13], [14]. They are defined by

$$
\begin{aligned}
\mathrm{PW}_{2 N-1}^{2 M-1}(t, \omega):= & 2 \sum_{k=-\infty}^{\infty} e^{-i 2 \omega k}\left|h_{N}(k)\right|^{2} \\
& \cdot \sum_{m=-\infty}^{\infty} g_{M}(m) X(t+m+k) \\
& \cdot X^{*}(t+m-k)
\end{aligned}
$$

where $h_{N}(k)$ and $g_{M}(m)$ again denote windows with $2 N-$ 1 , respectively $2 M-1$, nonzero values. This estimator has been called the smoothed pseudo-Wigner estimator if $M>1$. An estimator like this is a member of the general class of spectral estimators; it is defined by the weighting function

$$
\phi_{\mathrm{SPW}}(n, 2 k)=\left|h_{N}(k)\right|^{2} \cdot \sum_{m=-\infty}^{\infty} g_{M}(m) e^{-i n m} .
$$

Comparing $\phi_{\mathrm{SPW}}$ and $\phi_{\mathrm{SSTP}}$, it appears that $\phi_{\mathrm{SPW}}$ offers the advantage of being a separable weighting function, 
whereas $\phi_{\text {SSTP }}$ is governed by "uncertainty relations": $\phi_{\text {SSTP }}$ is defined via an ambiguity function. This results in a combined time-frequency smoothing, and therefore the performance in one direction can only be altered at the expense of an alteration of the performance in the other direction. The weighting function of the smoothed pseudoWigner estimator overcomes this difficulty: it allows an independent smoothing in both directions, time and frequency.

The moments of the smoothed pseudo-Wigner estimator can again be calculated by using the general expressions (16), (25), and (28) when taking care of the necessary conditions for the approximations used. The final results are

$$
\begin{gathered}
E\left\{\mathrm{PW}_{2 N-1}^{2 M-1}(t, \omega)\right\}=\frac{1}{2 \pi} \sum_{m=-\infty}^{\infty} g_{M}(m-t) \\
\int_{-\pi / 2}^{\pi / 2} W_{h}(0, \omega-\zeta) W(m, \zeta) d \zeta \\
C \sim \begin{cases}0, & \left|\omega_{1}-\omega_{2}\right| \geqq \pi \frac{M}{N} \\
2 W_{f_{t}}\left(\tau, \omega ; \phi_{2}\right), & \omega_{1}=\omega_{2}=\omega\end{cases} \\
\quad \operatorname{Var}\left\{\mathrm{PW}_{2 N-1}^{2 M-1}(t, \omega)\right\} \sim 2 W_{f t}\left(0, \omega ; \phi_{2}\right)
\end{gathered}
$$

with $\phi_{2}(n, k)=\left|G_{M}(n)\right|^{2}, G_{M}(n)$ defined by (36) and a normalization of

$$
\sum_{k=-\infty}^{\infty}\left|h_{N}(k)\right|^{4}=1
$$

In [13], these results have been obtained by directly evaluating (42).

Due to the separability of the weighting function $\phi_{\text {SPW }}$, the bias in (44) is now split up into two contributions which can be controlled separately. In particular, the unsmoothed pseudo-Wigner estimator $(M=1)$ has no bias in the time direction. A second advantage of the smoothed pseudo-'Wigner estimators consists of their noncorrelation in the frequency direction for an appropriate spacing: this is given by (45).

According to the marginal distribution (7), we have as an approximation of (46) (valid if $M$ is sufficiently large)

$$
\operatorname{Var}\left\{\mathrm{PW}_{2 N-1}^{2 M-1}(t, \omega)\right\} \sim \frac{2}{2 M-1} f_{t}^{2}(\omega) .
$$

Hence, the variance decreases with the order of $1 /(2 M-$ 1) as in the case of the smoothed short-time periodograms. Nevertheless, pseudo-Wigner estimators allow us look at the data giving a lot of detailed structures: this smoothing brings up only the same amount of loss of resolution as in the case of the smoothed short-time periodograms [cf. (41)], but the unsmoothed pseudo-Wigner estimator is superior to the unsmoothed short-time periodograms as shown by (34) and (44). By their flexibility due to the separable weighting function $\phi_{\mathrm{SPW}}$, they offer an interesting alternative to smoothed short-time periodograms when refined structures of the data are to be evaluated.

The major problem of nonstationary spectral analysis is then not the choice of an observation window as in the case of short-time periodograms, but only the estimation of the amount of smoothing over neighboring estimates where we have a tradeoff between variance reduction and loss of nonstationary properties. We have proposed elsewhere [13] an Akaike-type criterion as a solution, which works especially well in mixtures of signals and noise [30].

\section{F. Examples}

As a first example, we consider nonstationary white noise. This is a random signal $X(t)$ with autocovariance function

$$
K(t+k, t-k)=\sigma_{t}^{2} \delta(k), \quad \sigma_{t}^{2}>0 \text { for all } t .
$$

According to (4), its Wigner-Ville spectrum is given by

$$
W(t, \omega)=2 \sigma_{t}^{2} .
$$

Equation (50) depicts the Wigner-Ville spectrum of nonstationary white noise as the ordinary spectral density of a stationary white noise if $t$ is fixed.

If $\sigma_{t}^{2}$ is a sufficiently smooth function, $X(t)$ is a quasistationary signal. Then we can evaluate the variance of special spectral estimators. Let the spectral density $f_{i}(\omega)$ of the tangential stationary (analytic) process be given by

$$
f_{t}(\omega)=2 \sigma_{t}^{2}, \quad 0<\omega<\pi .
$$

We have then from (23) with $\phi \equiv 1$

$$
W_{f t}(\tau, \omega)= \begin{cases}2 \sigma_{t}^{4} \frac{1}{\tau} \sin 2 \tau \omega, & 0<\omega<\pi / 2 \\ 2 \sigma_{t}^{4} \frac{1}{\tau} \sin 2 \tau(\pi-\omega), & \pi / 2<\omega<\pi\end{cases}
$$

and, from (41) and (46),

$$
\begin{aligned}
\operatorname{Var}\left\{J_{2 N-1}^{2}(t, \omega)\right\} & \sim 4 \sigma_{t}^{4}, \quad 0<\omega<\pi \\
\operatorname{Var}\left\{\mathrm{PW}_{2 N-1}(t, \omega)\right\} & \sim \begin{cases}\frac{4 \sigma_{t}^{4}}{\pi} 2 \omega, & 0<\omega<\pi / 2 \\
\frac{4 \sigma_{t}^{4}}{\pi} 2(\pi-\omega), & \pi / 2<\omega<\pi .\end{cases}
\end{aligned}
$$


Equations (53) and (54) show that the variance of the pseudo-Wigner estimator depends on the frequency variable. It is always lower than that of the short-time periodograms other than at the frequency $\omega=\pi / 2$ where both variances are equal. form

As a second example, a multiplicative MA model of the $X(t)=g(t)(\epsilon(t)-\alpha \epsilon(t-2)), \quad 0<\alpha<1, g(t)>0$

is discussed. If $g(t) \equiv 1, X(t)$ is a stationary MA model with spectral density

$$
f(\omega)=\sigma_{\epsilon}^{2}\left|1-\alpha e^{-i 2 \omega}\right|^{2}
$$

where $\sigma_{\epsilon}^{2}$ is the variance of the Gaussian centered white noise $\epsilon(t)$. If $g(t)$ is any time-varying function, $X(t)$ is nonstationary and possesses an evolutionary time-varying spectrum in the sense of Tjøstheim and Mélard [6], [8]:

$$
f_{t}(\omega)=g^{2}(t) \sigma_{\epsilon}^{2}\left|1-\alpha e^{-i 2 \omega}\right|^{2}
$$

and a Wigner-Ville spectrum (based on the real signal only)

$$
\begin{aligned}
W(t, \omega)= & 2 \sigma_{\epsilon}^{2}\left\{g^{2}(t)\left(1+\alpha^{2}\right)\right. \\
& -2 \alpha g(t+1) g(t-1) \cos 2 \omega\} .
\end{aligned}
$$

Note that both spectra reduce to the ordinary stationary spectrum if $g(t)=1$, but clearly, both spectra differ. This causes problems if one wants to compare the numerical performances of general spectral estimators to estimates based on time-varying models: the theoretical spectra to be estimated by these two different classes of estimators are different if the signals are nonstationary.

\section{Numerical Implementation and Application of PSEUdo-Wigner Estimators}

\section{A. Numerical Implementation}

It has been shown in Section III that pseudo-Wigner estimators form a very attractive special class of general spectral estimators recommending its practical application. We shall therefore indicate the implementation of an adequate algorithm for its numerical realization.

We start with (42) and set at first $M=1$. When having in mind to apply FFT techniques, we set $\omega_{n}:=\pi(n / N)$ and we get

$$
\begin{gathered}
\mathrm{PW}_{2 N-1}\left(t, \omega_{n}\right)=2 \sum_{k=-N+1}^{N-1} e^{-i 2 k \pi(n / N)}\left|h_{N}(k)\right|^{2} \\
\cdot x(t+k) x *(t-k) .
\end{gathered}
$$

In (59), $x(t)$ denotes the analytical signal of the sampled realization of the random signal $X(t)$. We can split up the sum going from $-N+1$ to $N-1$ into two sums running from 0 to $N-1$. This results in

$$
\begin{aligned}
\mathrm{PW}_{2 N-1}\left(t, \omega_{n}\right)= & 2\left(2 \operatorname { R e } \left\{\sum_{k=0}^{N-1} e^{-i 2 k \pi(n / N)}\left|h_{N}(k)\right|^{2}\right.\right. \\
& \left.\left.x(t+k) x^{*}(t-k)\right\}-|x(t)|^{2}\right) .
\end{aligned}
$$

Hence, if $N$ is a power of two, we can calculate the pseudoWigner estimator over $2 N-1$ data points by one ordinary FFT over $N$ data points. This reduces the computational burden and allows an efficient evaluation of pseudoWigner estimates.

If the smoothed pseudo-Wigner $(M>1)$ is to be calculated, we only have to add a smoothing over neighbored pseudo-Wigner estimates just like in the case of smoothed short-time periodograms.

In many applications, one wants to perform a real-time analysis or at least a quasi-real-time one. This can also be done by the pseudo-Wigner estimator. In order to evaluate (60), we have first to calculate the analytic signal of a realvalued realization of $X(t)$ : this costs two FFT's, each over $2 N$ points in order to calculate the Hilbert transform. Then, we calculate (60) by one FFT over $N$ points. The time necessary to perform all these operations now dictates the temporal resolution we may obtain in a real-time application. Thus, the required frequency resolution and the rapidity of the device performing the FFT gives us the best temporal resolution we can obtain.

\section{B. Examples}

We shall now consider numerical examples dealing with signal + noise mixtures and with time-varying linear models. All computations presented here have been performed on a Floating Point Array Processor FPS-164 at RHRZ of the University of Bonn, Germany; the Hilbert transformer and the complex FFT of the library were used.

As a first example, Figs. 1 and 2 present pseudo-Wigner estimates for

$$
S_{1}(t)=\left\{\begin{array}{lr}
p(t) \sin \left\{\frac{2 \pi}{16} t+u(t-488) \pi\right\}, & 408<t<568 \\
0, & 0 \leqq t<409 \\
567<t<951
\end{array}\right.
$$




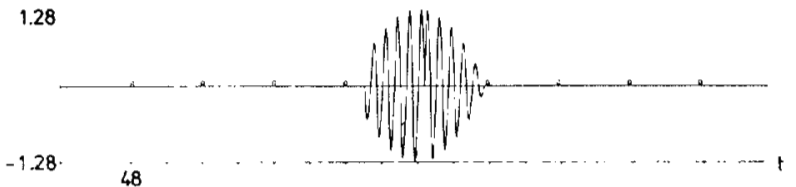

(a)

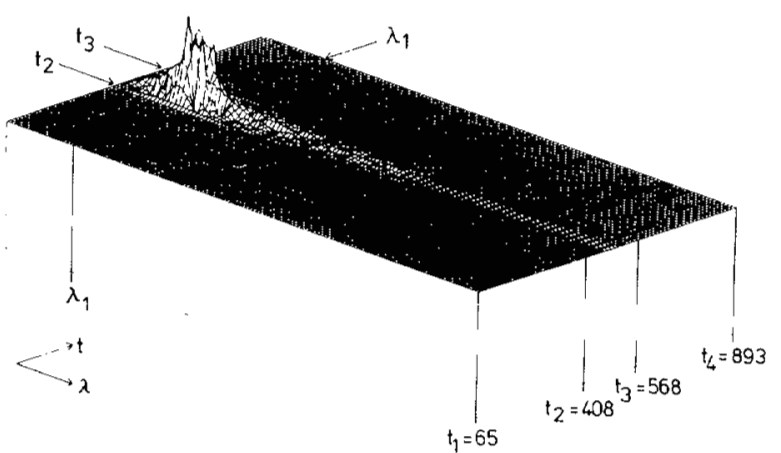

(b)

Fig. 1. Signal defined by (64) (a) and its pseudo-Wigner spectrum $(M=1)$ (b).

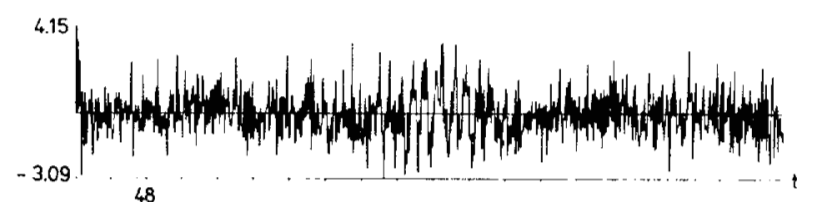

(a)

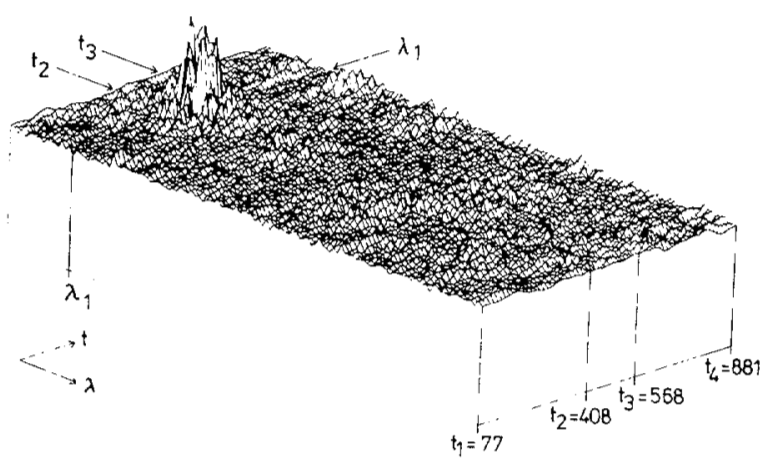

(b)

Fig. 2. Signal defined by (64) plus noise $\left(\mathrm{SNR}_{\max }=5 \mathrm{~dB}\right)$ (a) and its estimated pseudo-Wigner spectrum $(M=13)(\mathrm{b})$.

where $p(t)$ is a parabola taking its maximum at $t=488$; $u(t)$ is the unit step function. To get a noisy signal, we have blurred $S_{1}(t)$ with a Gaussian centered white noise such that the maximal SNR at $t=488$ is $5 \mathrm{~dB}$. Signals like this can be found in bioacoustical problems [26]. The observation window $h_{N}$ is a rectangular window with $N=$ 64 and a 128-point zero padding has been applied. Frequency and time increments are, respectively, $\Delta \omega=\pi /$ 128 and $\Delta t=12$. The time origin and the degree of smoothing of the pseudo-Wigner estimator are specified on each figure as $t_{1}$ and $M$.

As a second example, Fig. 3 gives a fourth-order AR model modulated by a Gaussian amplitude:

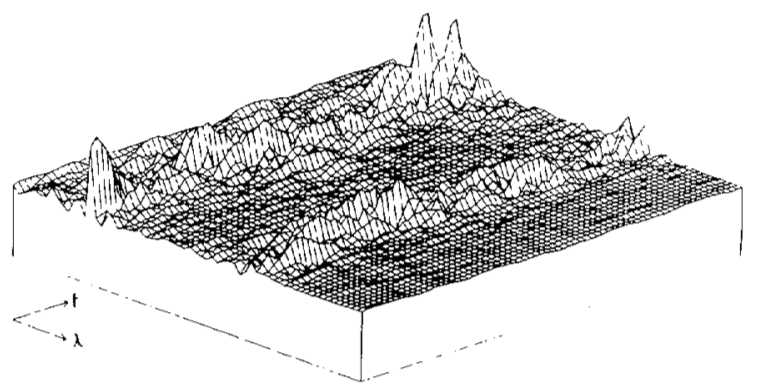

(a)

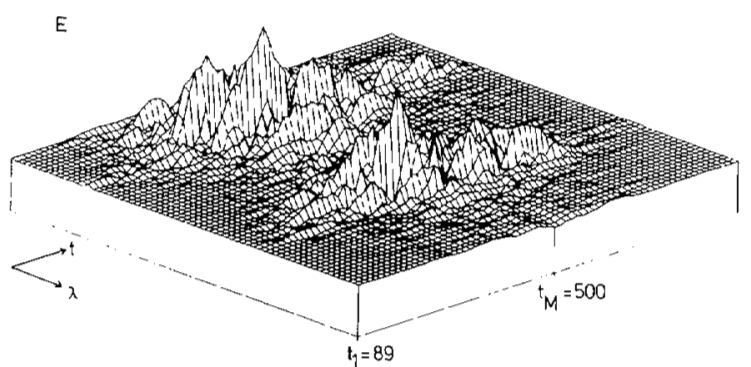

(b)

Fig. 3. Pseudo-Wigner estimates of the Wigner-Ville spectrum of $X(t)$ (a) and $S_{2}(t)($ b) defined by $(65)$. In both cases, $M=25$.

$$
\begin{aligned}
& X(t):= 0.5 X(t-1)-0.3 X(t-2)+0.4 X(t-3) \\
&-0.5 X(t-4)+\epsilon(t) \\
& S_{2}(t):= X(t) \exp \left(-\frac{1}{2}\left(\frac{t-500}{200}\right)^{2}\right) \\
& t=0, \cdots, 999
\end{aligned}
$$

where $\epsilon(t)$ is a centered normed Gaussian white noise. The same window and the same parameters as for Figs. 1 and 2 have been chosen (but without zero padding). It can be observed that the pseudo-Wigner estimators are sensible to refined signal structures, but this sensibility is not always adequate when analyzing time-varying models. Having inspected various examples, it seems that pseudoWigner estimators are a good device for pilot studies and for deciding whether an analysis by time-varying models is adequate for a given signal.

For further examples and numerical comparisons between smoothed short time periodograms and pseudoWigner estimators, we confer to [14], [21], [30], and [31].

\section{Application}

When studying the dynamics of biological oscillators, input-output experiments are often used. The interest mainly consists of the estimation of the ranges of synchronization of the biological oscillator to an external periodic cue [27]. In this kind of experiment, nonstationary phenomena occur if the external cue is switched on. Fig. 4 gives an example: the input signal is temperature [Fig. 4(a)]; the output signal is motility of a culture of unicellular green algae [Fig. 4(b)]. The smoothed pseudo-Wigner spectrum (Fig. 4(c), $M=13$ ) depicts the time-varying structure of the output signal. Motility gets slowly 
(a)

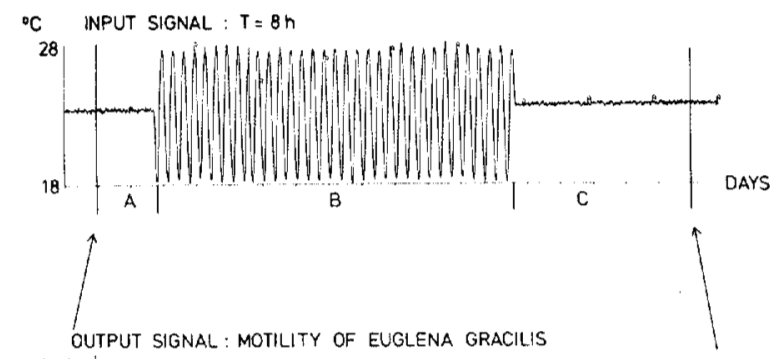

(b)

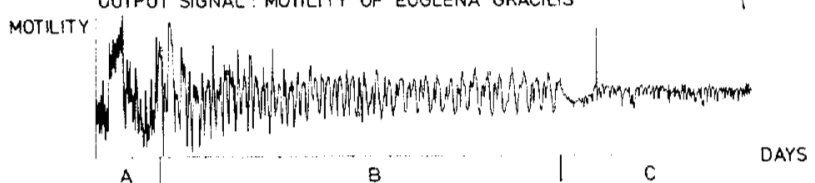

(c)

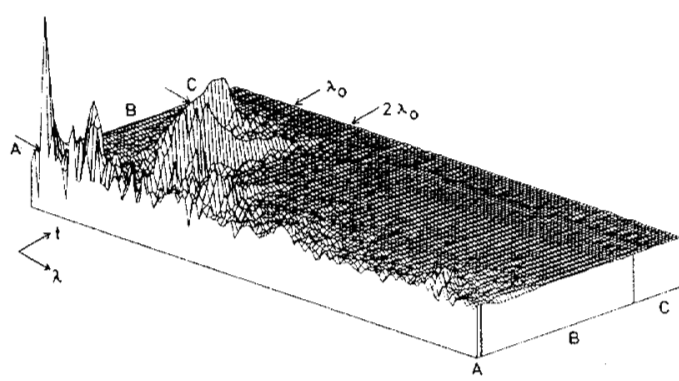

Fig. 4. Example of an input-output analysis for studying the dynamics of biological oscillators. (a) Input signal (temperature) with a frequency $\lambda_{0}$ $=0.125 \mathrm{~h}^{-1}$ in part (B). (b) Output signal (motility of a culture of unicellular green algae (Euglena gracilis) after detrending). The signal corresponds to the cutoff part of the input signal. (c) Wigner-Ville spectrum of the output signal estimated by a smoothed pseudo-Wigner estimator ( $M=13, N=128, N$ doubled by zero padding). The indicated sections $A, B$, and $C$ correspond to the same sections marked in parts (a) and (b) of this figure.

phase locked to the forcing temperature signal with frequency $\lambda_{0}=0.125 \mathrm{~h}^{-1}$. This is indicated by the increasing amplitude of the $\lambda_{0}$-frequency component. At the same time, higher harmonics of $\lambda_{0}$ show a decreasing amplitude [cf. the $2 \lambda_{0}$ component in Fig. 4(c)]. Higher harmonics are due to nonlinearities of the transfer system. The Wigner-Ville spectrum now shows the disappearance of these nonlinear properties of this system. When stopping the temperature periodicity [part (C)], there is no reappearance of the biological oscillator controlling motility under constant light and temperature conditions. According to the Wigner-Ville spectrum of part $(C)$, the output signal can be considered as white noise. Thus, the forcing periodic cue has altered the structure of biological system responsible for the generation of the oscillations. The biological interpretation of results like this will be published elsewhere [28].

\section{Conclusion}

Using the Wigner-Ville theory of conjoint time-frequency representations, we have defined a general class of spectral estimators of the Wigner-Ville spectrum of nonstationary random signals. As special cases, the class includes short-time periodograms and pseudo-Wigner estimators. The unifying framework allows the calculation of general expressions for the first and second moments of general spectral estimates. The results emphasize the ver- satility of pseudo-Wigner estimators: they are defined via a separable weighting function and, in contrast to shorttime periodograms, this permits more flexibility in a practical design. Pseudo-Wigner estimators are especially well equipped to visualize refined nonstationary structures, but if the spectral properties of the underlying process are more smooth, short-time periodograms are preferable, whereas their variance is often higher than that of comparable pseudo-Wigner estimators.

Furthermore, the separability of the weighting function defining the pseudo-Wigner estimator avoids the tradeoff between a required frequency resolution and the bias. The only critical quantity rests with the appropriate degree of smoothing between neighbored pseudo-Wigner estimates: this knowledge is necessary for deciding between the necessary reduction of variance and the smoothing out of nonstationary phenomena. As a solution, we have proposed elsewhere [13] an Akaike-type criterion for estimating the time of stationarity; numerical results have shown that our procedure works well, especially when signal plus noise mixtures are present [30].

\section{REFERENCES}

[1] M. B. Priestley, Spectral Analysis and Time Series. New York: Academic, $1982,890 \mathrm{pp}$.

[2] R. M. Loynes, "On the concept of the spectrum for nonstationary processes," J. Roy. Statist. Soc., ser. B, vol. 30, pp. 1-20, 1968.

[3] A. Blanc-Lapierre and B. Picinbono, "Remarques sur la notion de spectre instantané de puissance," Publ. Sci. Univ. Alger, ser. B, vol. 1, pp. 2-32, 1955.

[4] J. B. Allen and L. R. Rabiner, "A unified approach to short-time Fourier analysis and synthesis," Proc. IEEE, vol. 65, pp. 1558-1564, 1977.

[5] M. B. Priestley, "Evolutionary spectra and non-stationary processes," J. Roy. Statist. Soc., ser. B, vol. 27, pp. 204-229, 1965.

[6] G. Mélard, "Propriétés du spectre évolutif d'un processus non-stationnaire," Ann. Inst. H. Poincaré, sec. B, vol. 14, no. 4, pp. 411$424,1978$.

[7] Y. Grenier, "Time dependent ARMA modeling of nonstationary signals," IEEE Trans. Acoust., Speech, Signal Processing, vol. ASSP31, pp. 899-911, Aug. 1983.

[8] D. Tjøstheim, "Spectral generating operators for non-stationary processes." Adv. Appl. Prob., vol. 8, pp. 831-846, 1976.

[9] T. A. C. M. Claasen and W. F. G. Mecklenbräuker, "The Wigner distribution: A tool for time frequency signal analysis," Philips J. Res., vol. 35 , pp. 217-250, 276-300, 372-389, 1980.

[10] P. Flandrin and B. Escudié, "Time and frequency representation of finite energy signals: A physical property as a result of an Hilbertian condition," Signal Processing, vol. 2, no. 2, pp. 93-100, 1980.

[11] W. Martin, "Time-frequency analysis of random signals," in Proc. IEEE Int. Conf. Acoust., Speech, Signal Processing, Paris, France, 1982 , pp. $1325-1328$

[12] P. Flandrin and W. Martin, "Sur les conditions physiques assurant l'unicité de la représentation de Wigner-Ville comme représentation temps-fréquence," in $9^{\text {imc }}$ Coll. GRETSI, Nice, France, 1983, pp. 4349.

[13] W. Martin and P. Flandrin, "Analysis of non-stationary processes: Short-time periodograms versus a pseudo-Wigner estimator," in $\mathrm{H}$. Schüssler, Ed., EUSIPCO-83. Amsterdam: North-Holland, 1983, pp. 455-458.

[14] P. Flandrin and W. Martin, "Pseudo-Wigner estimators for the anal ysis of nonstationary processes," in Proc. IEEE ASSP Spectrum Est. Workshop 1I, Tampa, FL, 1983, pp. 181-185.

[15] W. Martin, "Time-frequency analysis of non-stationary processes," presented at the IEEE Int. Symp. Inform. Theory, Les Arcs, 1982.

[16] A. Blanc-Lapierre and B. Picinbono, Fonctions Aléatoires. Paris: Masson, 1981

[17] E. P. Wigner, "On the quantum correction for thermodynamic equilibrium," Phys. Rev., vol. 40, pp. 749-759, 1932. 
[18] J. Ville, "Théorie et applications de la notion de signal analytique," Cables et Trans., vol. 2, no. 1, pp. 61-74, 1948.

[19] W. D. Mark, "Spectral analysis of the convolution and filtering of non-stationary stochastic processes," J. Sound Vib., vol. 11, pp. 19$63,1970$.

[20] J. S. Bendat and A. G. Piersol, Measurement and Analysis of Random Data. New York: Wiley, 1966, p. 364.

[21] P. Flandrin, "Some features of time-frequency representations of multicomponent signals," in Proc. IEEE Int. Conf. Acoust., Speech, Signal Processing, San Diego, CA, 1984, pp. 41B4.1-41B4.4.

[22] B. Picinbono and W. Martin, "Représentation des signaux par amplitude et phase instantanées," Ann. Télécommun., vol. 38, no. 5-6, pp. $179-190,1983$.

[23] P. Flandrin and W. Martin, "A general class of estimators for the Wigner-Ville spectrum of non-stationary processes," in Lecture Notes in Control and Information Sciences, vol. 62. Berlin: Springer-Verlag, 1984, pp. 15-23.

[24] L. Cohen, "Generalized phase-space distribution functions," J. Math. Phys., vol. 7, pp. 781-786, 1966.

[25] B. Escudié and J. Gréa, "Sur une formulation générale de la représentation en temps et en fréquence dans l'analyse des signaux d'énergie finie," C. R. Acad. Sci. Paris, ser. A, vol. 283, pp. 1049-1051, 1976.

[26] R. H. Backus and W. E. Schevill, "Physeter clicks," in Whales, Dot phins, and Pórpoises, K. S. Norris, Ed. Berkeley and Los Angeles, CA: Univ. California Press, 1966, pp. 510-528.

[27] J. Aschoff and H. Pohl, "Phase relations between a circadian rhythm and its Zeitgeber within the range of entrainment," Naturwiss, vol. 65 , pp. 80-84, 1978.

[28] R. Jörres, W. Martin, and K. Brinkmann, "Identification of the temperature masking of the circadian system of Euglena Gracilis," in R. Trappl, Ed., Cybernetics and Systems Research II. Amsterdam: Elsevier, North-Holland, 1984, pp. 293-297.

[29] T. A. C. M. Claasen and W. F. G. Mecklenbräuker, "On the time-frequency discrimination of energy distributions: Can they look sharper than Heisenberg?," in Proc. IEEE Int. Conf. Acoust., Speech, Signal Processing, San Diego, CA, 1984, pp. 41B7.1-41B7.4.

[30] W. Martin and P. Flandrin, "Detection of changes of signal structures by using the Wigner-Ville spectrum," Signal Processing, vol. 8, no. 2, pp. 215-233, 1985.
[31] W. Martin and K. Krüger-Alef, "Application of the Wigner-Ville spectrum to a class of bio-acoustical signals blurred by noise," in Proc. Airborne Animal Sonar Syst. Colloq., Lyon, France, 1985.

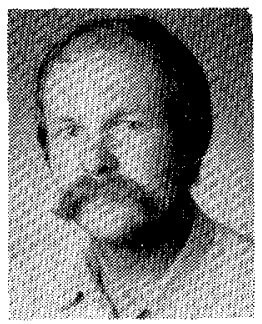

Wolfgang Martin was born in Siegburg, Germany in 1949. He received the Diploma in Mathematics from the University of Bonn, Germany, in 1973, the $\mathrm{Ph} . \mathrm{D}$. degree in applications of nonstationary processes to biological problems.

From 1981-1982 he was on leave at the Laboratoire des Signaux et Systèmes at Gif-sur-Yvette, France. In 1984 he researched habilitation for biometrics and signal theory at the University of Bonn.

Dr. Martin is member of EURASIP, GMDS, and the International Society of Biometrics.

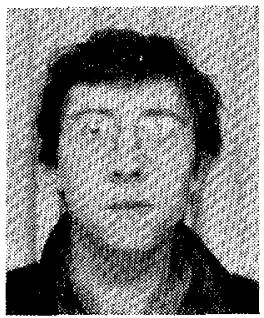

Patrick Flandrin was born in Bron, Rhône, France in 1955. He received the Ingénieur degree from the Institut de Chimie et Physique Industrielles de Lyon, Lyon, France, in 1978, the Docteur Ingénieur degree from Institut National Polytechnique de Grenoble in 1982

He joined the Centre National de la Recherche Scientifique in 1982. His principal interests are in nonstationary signal processing, relationships between signal theory and quantum mechanics, and the study of animal sonar systems.

Dr. Flandrin is a member of EURASIP. 